\title{
The Temperature Behavior of Resonant and Non-resonant Microwave Absorption in Ni-Zn Ferrites
}

\author{
Raúl Valenzuela \\ Departamento de Materiales Metálicos y Cerámicos, \\ Instituto de Investigaciones en Materiales, \\ México
}

\section{Introduction}

The magnetic response of $\mathrm{Ni}-\mathrm{Zn}$ ferrites at microwave frequencies has been recently investigated by means of resonance techniques, by several authors. In this chapter, we present a review of recent results obtained on the resonant microwave absorption (electron paramagnetic resonance, EPR, and ferromagnetic resonance, FMR) in the X-band $(9.5 \mathrm{GHz})$, of polycrystalline Ni-Zn ferrites $\left(\mathrm{Zn}_{\mathrm{x}} \mathrm{Ni}_{1-\mathrm{x}} \mathrm{Fe}_{2} \mathrm{O}_{4}\right)$ for several temperature ranges. We begin at high temperatures in the paramagnetic state $\left(T>T_{C}\right.$, where $T_{C}$ is the Curie point); as temperature decreases, the onset of magnetic ordering is investigated, with its effects on the main FMR parameters. When experiments are carefully carried out, magnetic transitions can be detected as critical points in plots of the thermal behavior of the resonance line width.

We investigate also the behavior of nonresonant properties by means of the low-field microwave absorption (LFMA). This absorption, which occurs at applied fields of the same order of magnitude than the anisotropy field, $H_{\mathrm{K}}$, of the sample, is providing valuable information concerning the magnetization processes. LFMA is typically measured in the $-1 \mathrm{kOe}<H_{\mathrm{DC}}<+1 \mathrm{kOe}$ field range. LFMA is associated with the nonresonant microwave absorption occurring during the magnetization processes from the unmagnetized state up to the approach to saturation. We provide here a short review of this particular measuring technique. Then, we propose to begin the study of LFMA in $\mathrm{Ni}-\mathrm{Zn}$ ferrites also by decreasing the measuring temperature from the Curie transition. Clearly, LFMA is absent at $T>T_{\mathrm{C}}$ since it depends on the magnetization processes in the ordered phase. For the $200 \mathrm{~K}<T<T_{\mathrm{C}}$ temperature range, a direct comparison of the anisotropy field calculated from LFMA and a calculation by using results of a direct measurement of $H_{\mathrm{K}}$ on a ferrite single crystal. A very good agreement is obtained, thus confirming that LFMA is strongly dependent of the total anisotropy (magnetocrystalline, magnetoelastic and shape anisotropies) of the sample.

We use as well a novel nonresonant microwave absorption technique known as magnetically modulated microwave absorption spectroscopy, MAMMAS. This technique is particularly well adapted to detect phase transitions of many types, as it is based on the change of microwave absorption regime during a change of crystalline, magnetic or electronic structure. MAMMAS is briefly described and applied to Ni-Zn ferrites. 


\section{Ferrites}

Ferrites, also known as magnetic ceramics, are a very well established group of magnetic materials (Valenzuela, 2005a). Ferrites possess three different crystal structures: spinels, garnets, which belong to cubic systems, and hexagonal, which can be considered as derived from magnetoplumbite. In this review, the focus will be on spinel ferrites, and in particular on the Ni-Zn "family" which will be taken as an example. A brief review of crystal structure, magnetic structure and magnetic properties of these ferrites is given.

\subsection{Spinel structure}

The spinel structure is a cubic structure extremely stable, with a dominant ionic character. In addition to charge compensation, the cation/anion ratio is $3 / 4$. More than 140 oxides and 80 sulphides have been systematically studied (Hill \& al 1979). Most of the commercially important spinels are synthetic, but the most important and probably the oldest one with practical applications, magnetite, $\mathrm{Fe}_{3} \mathrm{O}_{4}$, is a natural oxide. Magnetite has also the remarkable feature of the simultaneous presence of ferrous $\left(\mathrm{Fe}^{2+}\right)$ and ferric $\left(\mathrm{Fe}^{3+}\right)$ iron on equivalent crystal sites, which provides unusual electrical and magnetic properties. In addition to the 2,3 spinels (2,3 refers to divalent and trivalent cations, respectively), formed by a combination of one divalent and two trivalent cations to balance the 8 negative charges provided by the oxygen in the formula $\mathrm{D}^{+2} \mathrm{~T}^{+3}{ }_{2} \mathrm{O}^{-2} 4$, there are other combinations with spinel structure, which provide 3 cations with a total valency of 8 , such as $2,4\left(\mathrm{Co}_{2} \mathrm{GeO}_{4}\right)$, 1,3,4 $\left(\mathrm{LiFeTiO}_{4}\right), 1,3\left(\mathrm{Li}_{0.5} \mathrm{Fe}_{2.5} \mathrm{O}_{4}\right), 1,2,5\left(\mathrm{LiNiVO}_{4}\right)$, and 1,6 $\left(\mathrm{Na}_{2} \mathrm{WO}_{4}\right)$ spinels.

The crystal structure, belonging to the $\mathrm{Fd} 3 \mathrm{~m}$ space group, can be described as a close-packed (fcc) arrangement of oxygens, which includes tetrahedral and octahedral interstitial sites. Onehalf of the interstitial octahedral sites and one-eighth of the tetrahedral sites are occupied by cations. They are known also as " $\mathrm{A}$ " sites (tetrahedral) and " $\mathrm{B}$ " sites (octahedral).

The unit cell is formed by eight formula units $\mathrm{AB}_{2} \mathrm{O}_{4}$, with eight $\mathrm{A}$ sites, $16 \mathrm{~B}$ sites and 32 oxygen. This unit cell can be divided into octants of edge $a / 2$ ( $a=$ unit cell parameter) for a better view of the two sites, Fig. 2.1. In this representation, a tetrahedral cation is taken as the origin of the cell. The nearest neighbors of both sites are illustrated in Fig. 2.2.

When divalent cations occupy the A sites and trivalent cations enter the B sites, the spinel is known as having a "normal" cation distribution. This arrangement can be represented as $\left(\mathrm{D}^{+2}\right)\left[\mathrm{T}^{3+}{ }_{2}\right]$. A variant of this structure is the "inverse" spinel, where A sites contain a trivalent cation, while $B$ sites contain the divalent and the remaining trivalent cation, $\left(\mathrm{T}^{3+}\right)$ $\left[\mathrm{D}^{2+} \mathrm{T}^{3+}\right]$. In some cases, an intermediate distribution can be achieved by playing with thermal treatments, leading to $\left(\mathrm{D}_{1-\delta} \mathrm{T}_{\delta}\right)\left[\mathrm{D}_{\delta} \mathrm{T}_{2-\delta}\right]$, where $\delta$ is the "degree of inversion". The distribution of cations on the two spinel sites depend on a complex interplay of cation radius, electrostatic energy, crystal field energy, and polarization effects (covalency contribution, for instance).

A remarkable feature of stability of spinel structure is that it can form an extremely large variety of total solid solutions. Some conditions apply; first, electrical neutrality, i.e., the addition of the charge of all cations should balance oxygen total charge (-8 for a formula); second, the ratio of cations/oxygen should remain $3 / 4$, and finally, there should be relatively small differences between cation radii. In solid solutions, composition can be changed on a continuous basis, leading also to continuous variations in the physical properties. This allows a very precise tailoring of magnetic properties, which is a major advantage for any application. Divalent cation in the 2,3 spinel formula can be formed by any combination of 

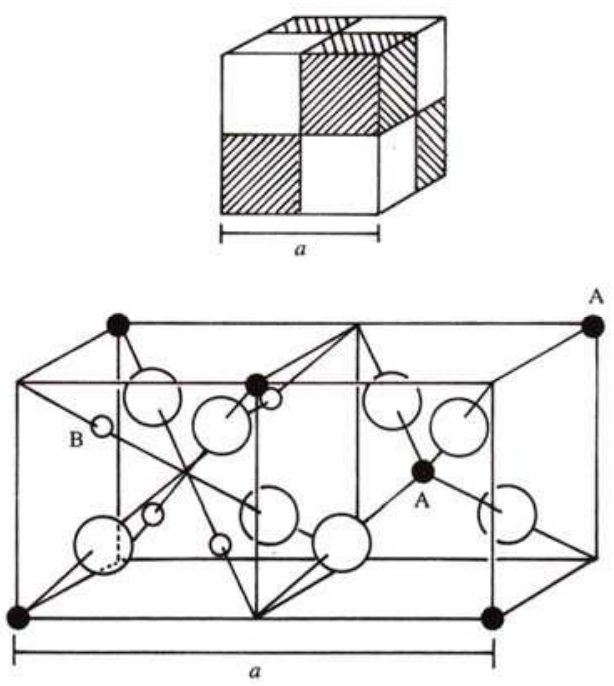

Fig. 2.1. Unit cell of the spinel structure. Cations on A sites are represented by small black circles, cations on octahedral B sites by small open circles, and large circles are oxygens. The unit cell parameter is $a$.

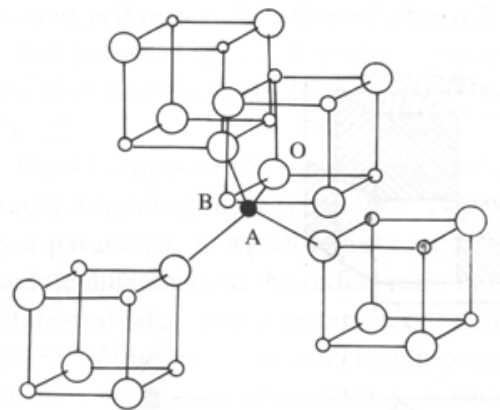

(a)

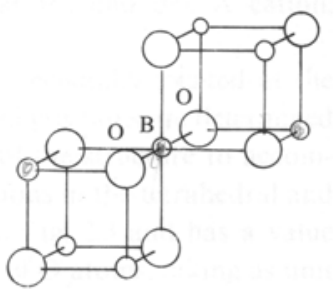

(b)

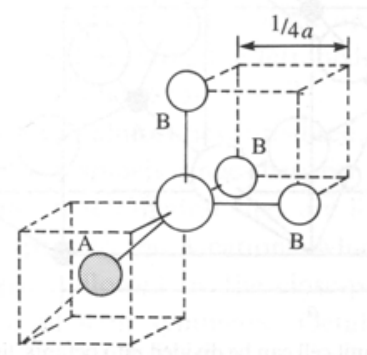

(c)

Fig. 2.2. Nearest neighbors of a) A site, b) B site and c) oxygen site. 
divalent $\mathrm{Ni}^{2+}, \mathrm{Co}^{2+}, \mathrm{Mn}^{2+}, \mathrm{Fe}^{2+}, \mathrm{Cu}^{2+}, \mathrm{Zn}^{2+}, \mathrm{Cd}^{2+}, \mathrm{Mg}^{2+}, \mathrm{Ca}^{2+}$. Ferric ions can also be substituted, or combined with $\mathrm{Al}^{3+}, \mathrm{V}^{3+}, \mathrm{Cr}^{3+}, \mathrm{Mn}^{3+}, \mathrm{Ga}^{3+}, \mathrm{In}^{3+}$, etc. One of the most interesting and representative solid solution is Ni-Zn ferrites, with formula $\mathrm{Ni}_{1-x} \mathrm{Zn}_{\mathrm{x}} \mathrm{Fe}_{2} \mathrm{O}_{4}$, with $0 \leq x \leq 1.0$ (Ravindranathan \& Patil, 1987).

\subsection{Nickel-zinc ferrites}

In spite of having a large cation radius, $\mathrm{Zn}^{2+}$ has a strong preference for A sites, which are smaller than $B$ sites. Ferric ions manifest no preference for A or B sites. Therefore, zinc ferrite $\mathrm{ZnFe}_{2} \mathrm{O}_{4}$ is a normal spinel. In contrast, divalent nickel shows a strong tendency to occupy $\mathrm{B}$ sites. This means that nickel ferrite, $\mathrm{NiFe}_{2} \mathrm{O}_{4}$ tends to be an inverse spinel. Ni-Zn solid solutions (when prepared by solid state reaction with a slow cooling from the sintering temperature) exhibit therefore a cation distribution which is normal with respect to $\mathrm{Zn}$, and inverse for $\mathrm{Ni}$. This means that $\mathrm{Zn}$ will occupy A sites (with ferric ions completing the "filling" of A sites), while nickel and the remaining ferric cations share B sites:

$$
\left(\mathrm{Zn}_{\mathrm{x}} \mathrm{Fe}_{1-\mathrm{x}}\right)\left[\mathrm{Ni}_{1-\mathrm{x}} \mathrm{Fe}_{1+\mathrm{x}}\right]
$$

The cell parameter, Fig. 2.3a, shows a linear dependence with composition $x$. Since $\mathrm{Zn}^{2+}$ is a relatively large cation occupying the small A sites, the cell parameter increases with $\mathrm{Zn}$ content. The Curie temperature exhibits a strong decrease with zinc concentration, Fig. 2.3b. For $x=1$, zinc ferrite $\left(\mathrm{ZnFe}_{2} \mathrm{O}_{4}\right)$ manifests an antiferromagnetic behavior with a Néel temperature of $9 \mathrm{~K}$. While the increase in cell parameter is quite linear, the decrease in $T_{\mathrm{C}}$ is more rapid. This fact can be understood by recalling that as $\mathrm{Zn}$ content increases, in addition to the expansion of the unit cell (and therefore, cations become far apart), there is an effect of dilution, since $\mathrm{Zn}$ cations are diamagnetic. However, there is also a change in magnetic structure, since for the very high content of $\mathrm{Zn}$, the ferrite changes from ferrimagnetic (with a high $T_{C}=858 \mathrm{~K}$ for $x=0$ ), to an antiferromagnetic arrangement with a very low Néel temperature. This result will be briefly discussed below.

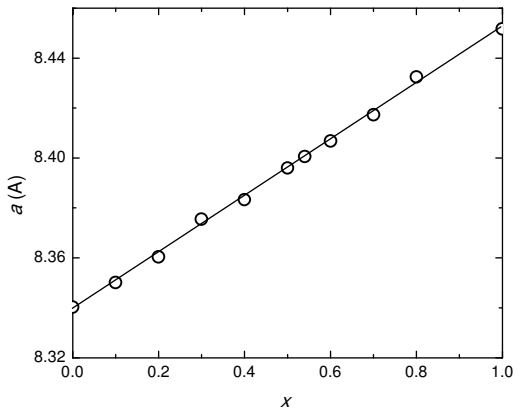

(a)

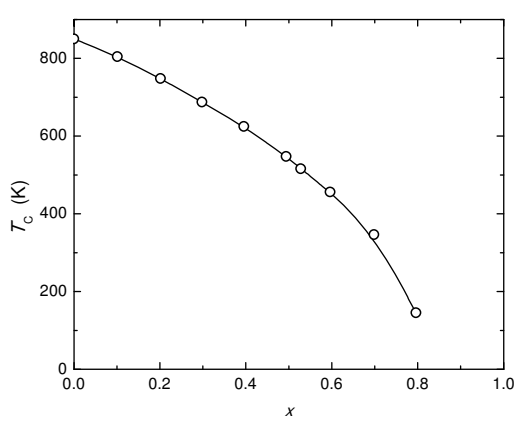

(b)

Fig. 2.3. Variation in the cell parameter, (a), and the Curie transition, (b), both as a function of Zn content $x$ (Adapted from Valenzuela, 2005a).

The ferrimagnetic order in ferrites is the result of superexchange interactions. The $3 \mathrm{~d}$ unpaired spins of transition metals exhibit an antiparallel arrangement which occurs through anions, as schematically shown in Fig. 2.4. This interaction takes place by means of 
p orbitals of oxygen. Since p orbitals are linear, this interaction sensitively depends not only on the distance between cations and anion, but also on the angle between them. It is expected to be a maximum for a $180^{\circ}$ angle. The first discussion on superexchange interactions was proposed by Anderson (1959).

The main superexchange interactions in spinels are the A-O-B and the B-O-B interactions. The former takes place between a cation in an A site, which becomes antiparallel to cations on the nearest $\mathrm{B}$ site. The latter consists on the antiparallel arrangement between two cations on neighboring $\mathrm{B}$ sites. The A-O-B interaction is expected to be significantly stronger than the B-O-B one, since the angle between these sites is close to $180^{\circ}$ (see Fig. 2.2 (c)); the B-O-B geometry involves a $90^{\circ}$ angle, quite different from the linear geometry of p orbitals.

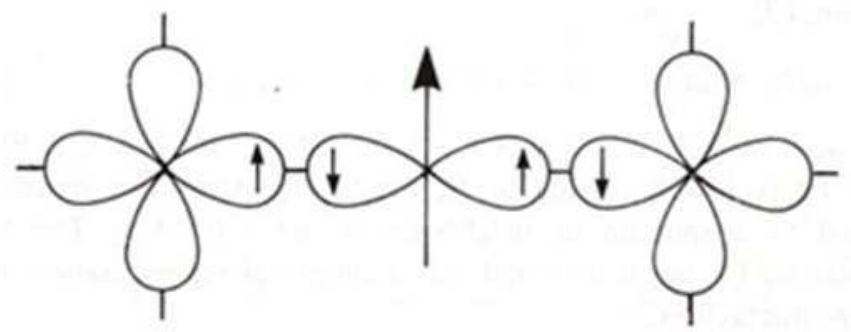

Fig. 2.4. Schematical representation of the superechange interactions in oxides. The spins in the unfilled $3 \mathrm{~d}$ orbitals of transitions metals, on the sides, can interact with cation nearest neighbors through the $2 \mathrm{p}$ oxygen orbitals, in the center. This interaction can be extremely strong, leading to high Curie temperatures.

For $x=0$, the cation distribution is as follows: $(\mathrm{Fe})[\mathrm{NiFe}]$. By assuming that A-O-B interaction is dominant, the iron in the A site will be aligned in an antiparallel direction with respect to spins of cations on B sites. If we simplify the magnetic structure of Fig. 2.2 (c) and represent one A site and two $B$ sites around an oxygen anion (in the basic formula, the ratio of $A$ to $B$ sites is $1 / 2$ ), and if all of them are assumed to be on the same plane, we can draw a cartoon like the one on Fig. 2.3. Nickel ferrite, with one $\mathrm{Fe}^{3+}$ on the A site, the other one on a $\mathrm{B}$ site and the Ni on the other B site should have a magnetic structure like the one in Fig. 2.5 (a). The interaction $\mathrm{Fe}(\mathrm{A})-\mathrm{O}-\mathrm{Fe}(\mathrm{B})$ is among the strongest in spinels, as $\mathrm{Fe}^{3+}$ has a $3 \mathrm{~d}$ orbital half-filled and the angle between sites is close to $180^{\circ}$. Accordingly, the Curie temperature is maximum for this family ( $858 \mathrm{~K}$ ), and it has the same value for most inverse spinels (such as $\mathrm{CoFe}_{2} \mathrm{O}_{4}$, for instance).

For zinc ferrite $(x=1)$, the site occupancy is: $(\mathrm{Zn})\left[\mathrm{Fe}_{2}\right]$. The A site contains only $\mathrm{Zn}$ ions (with no magnetic moment) and therefore the only interaction in the system is B-O-B. Irons on both B sites become antiparallel and the ferrite is antiferromagnetic, with a Néel temperature of $9 \mathrm{~K}$. This low value of superexchange interaction is explained mostly by the angle between interacting cations $\left(90^{\circ}\right)$, and also by the expansion of the unit cell, as a consequence of the larger size of $\mathrm{Zn}$ cations [Fig. 2.3.(a)]. For compositions in the $0.5<x<$ 0.8 range, with a distribution: $\left(\mathrm{Zn}_{x} \mathrm{Fe}_{1-\mathrm{x}}\right)\left[\mathrm{Ni}_{1-\mathrm{x}} \mathrm{Fe}_{1+\mathrm{x}}\right]$, where both interactions become comparable, the magnetic structure can be represented by a triangular arrangement known as the Yafet-Kittel structure, first proposed by these authors (Yafet and Kittel 1952). 


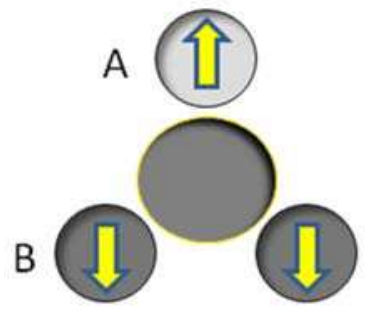

(a)

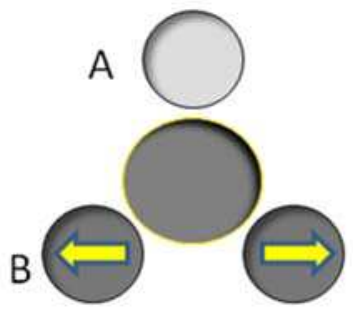

(b)

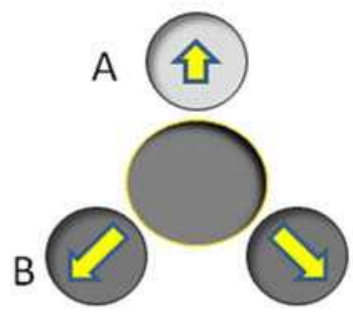

(c)

Fig. 2.5. Simplified representation of an A and two B sites around an oxygen. Arrows represent the spins as they can be expected for (a) nickel ferrite $(x=0)$, (b) zinc ferrite $(x=1)$, and (c) a composition rich in $\mathrm{Zn}(0.5<x<0.8)$.

A plot of saturation magnetization (at low temperatures) as a function of the composition starts at $\sigma_{\mathrm{s}} \sim 2.33 \mathrm{Bohr}$ magneton/formula unit, since the ferric cations are in opposition (Fig. 2.5 (a)) leaving only the nickel magnetic moment as a result, as shown in Fig. 2.6. If the A-O-B interaction were dominant on all the composition range, the total magnetic moment would exhibit an increase with $x$ up to a value of 10 Bohr magnetons for $x=1$ (broken line in Fig. 2.6), a condition with all A sites occupied by Zn (with no magnetic moment) and both $B$ sites with $\mathrm{Fe}$, and spins in a parallel orientation. But the weakening of this interaction results in the competition of B-O-B interaction, leading to the antiparallel arrangement on sites B, with the variations in saturation magnetization illustrated in Fig. 2.6.

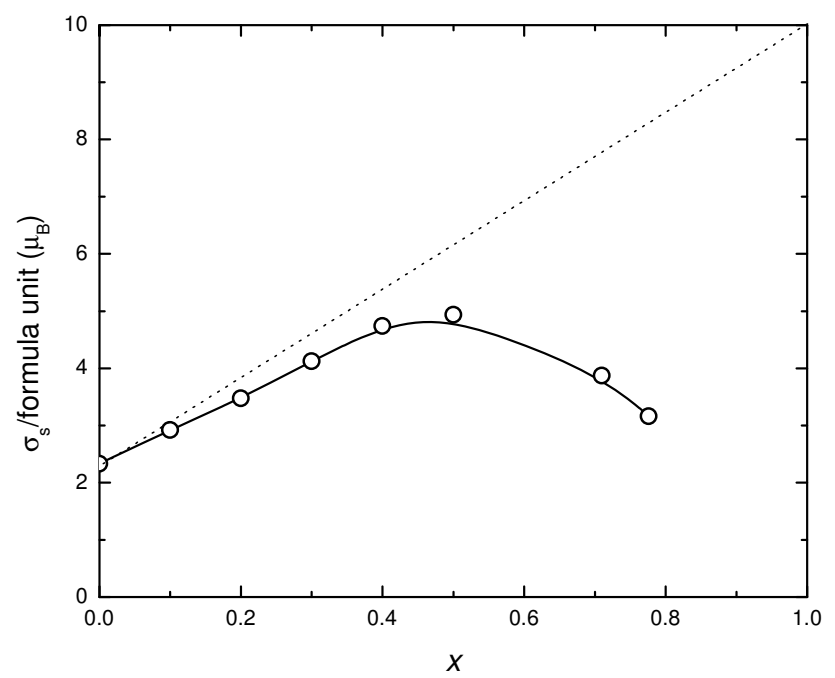

Fig. 2.6. Behavior of saturation magnetization of $\mathrm{Ni}_{1-x} \mathrm{Zn}_{x} \mathrm{Fe}_{2} \mathrm{O}_{4}$ ferrites at very low temperature, as a function of $\mathrm{Zn}$ content.

After many years, NiZn ferrites remain as an excellent system to study magnetic properties of solids. 


\section{Microwave absorption}

Microwave absorption has become a very powerful investigation and characterization tool in the study of magnetic materials, both in the paramagnetic, disordered state (electron paramagnetic resonance, EPR) and the ferri or ferromagnetic, ordered phase (electron ferromagnetic resonance, FMR) (see, for instance, Kittel 2005, Pilbrow 1990). The radiation emerging from interaction with a solid possesses changes (with respect to the incident radiation) that in principle, allow deducing the structural and magnetic properties of the material. To simplify, we can consider the interaction of a spin with a constant magnetic field. If the magnetic moment is originated only by the spin,

$$
\boldsymbol{\mu}=\mathrm{g} \mu_{\mathrm{B}} \boldsymbol{S}=\gamma \mathbf{S}
$$

where $\mathrm{g}$ is the gyromagnetic factor (in general depending on $\mathbf{L}$ and $\mathbf{S}$, the quantum mechanical numbers of orbital and spin momenta), $\gamma$ is the total gyromagnetic ratio. In an external field, $\mathbf{H}_{0}=H_{0} \mu_{\mathrm{z}}$, and energy is expressed as:

$$
E=-\mu \cdot \mathbf{H}=-\mu_{\mathrm{z}} H=-\gamma m_{\mathrm{s}} \hbar H
$$

with the spin $m_{\mathrm{s}}= \pm 1 / 2$, corresponding to the two orientation of the magnetic moment, i.e., parallel $\left(m_{\mathrm{S}}=-1 / 2\right)$, or antiparallel $\left(m_{\mathrm{S}}=+1 / 2\right)$ to the magnetic field; the population of both levels is given by the Boltzmann statistics,

$$
f=N^{+} / N^{-}=\exp \left\{-\Delta E / \mathrm{k}_{\mathrm{B}} T\right\}
$$

where $\mathrm{N}=\mathrm{N}^{+}+\mathrm{N}^{-}$is the total population of atoms with spin parallel $\left(\mathrm{N}^{-}\right)$and antiparallel $\left(N^{+}\right)$to the magnetic field, $\mathrm{k}_{\mathrm{B}}$ is the Boltzmann constant, and $-\Delta E$ the energy difference between the two levels. The net magnetic moment per atom is then:

$$
\mu_{\mathrm{z}}=\left(\mathrm{g} \mu_{\mathrm{B}} / 2\right)\left[\left(N^{+}+N^{-}\right) / N\right]=\left(g \mu_{\mathrm{B}} / 2\right)[(1-f) /(1+f)]
$$

A series expansion of (3.4) for not so low temperatures $\left(\mathrm{k}_{\mathrm{B}} T \gg \Delta E\right)$ leads to the Curie law,

$$
\left\langle\mu_{\mathrm{z}}\right\rangle=\left(\mathrm{g} \mu_{\mathrm{B}} / 3\right)\left(\Delta E / \mathrm{k}_{\mathrm{B}} T\right)=C H / T
$$

with $C=g \mu_{\mathrm{B}}{ }^{2} / 3 \mathrm{k}_{\mathrm{B}}$. It is possible to induce transitions between the two spin states by application of electromagnetic radiation of the relevant frequency, which satisfies the Bohr condition,

$$
\begin{aligned}
& \Delta E=\gamma \hbar H=\hbar \omega \\
& \omega=\gamma H
\end{aligned}
$$

This shows the resonance conditions. Equation (3.6) is also known as the Larmor resonance condition.

In the case of magnetic materials with a spontaneous magnetization (ferri and ferromagnetic materials), $H$ includes the internal field, in most cases leading to a lower external field needed to attain the resonance conditions. Both EPR and FMR have been used to investigate a wide variety of materials such as ferrites (Montiel et al 2004, Wu et al 2006) and amorphous alloys (Valenzuela et al 2005b, Montiel et al 2006).

In addition to these methods, nonresonant microwave absorption, or low field microwave absorption (LFMA) has been observed in many materials, such as amorphous metallic thin 
films (Rivoire \& Suran 1995), amorphous ribbons (Medina et al 1999), glass coated amorphous microwires (Chiriac et al 2000), ferrites (Montiel et al 2004), multilayer thin films (de Cos et al 2007). LFMA is strongly associated with magnetic order since in all cases it is present only below the transition temperature between the paramagnetic-ferrimagnetic (or para-ferromagnetic) phases. LFMA has also shown to be sensitive to mechanical stresses (Montiel et al 2006). In this chapter, we show that LFMA can also be used to detect changes in the magnetic structure. From the experimental point of view, LFMA needs an accurate measurement of the magnetic field for low fields, and the possibility to reverse the field, i.e., typically in the $-1000<H<+1000$ Oe. This can be challenging in the case of large electromagnets, which tend to keep a non negligible remanent field.

Another nonresonant method recently proposed for the investigation of magnetic transition is the method known as magnetically modulated microwave absorption spectroscopy (MAMMAS) (Alvarez \& Zamorano 2004, Alvarez et al 2007), which is based on a simple idea: the nonresonant microwave absorption regime in a given material changes when a phase transition occurs. Since the microwave absorption depends on the wide definition of structure (crystalline, electronic, magnetic, etc.), virtually any phase change can be detected, with the significant advantage that microwave absorption is extremely sensitive. Experimentally, the sample is subjected to a low magnetic field (clearly lower than the resonance field in the temperature range), and the microwave absorption is measured as the sample temperature is slowly varied. Phase transitions appear typically as a minimum in a $\mathrm{d} P / \mathrm{d} H$ vs $T$ plot.

\section{Microwave absorption in ferrites}

In this Section, we discuss the microwave absorption of polycrystalline $\mathrm{Ni}-\mathrm{Zn}$ ferrites as a function of measuring temperature. The resonant mode is first considered. The description and analysis of these properties is quite useful, as NiZn ferrites offer a wide variety of magnetic structures and phenomena. The study of non resonant absorption also sheds light on magnetic structure phenomena of ferrites.

\subsection{High temperatures $\left(T>T_{C}\right)$}

By "high temperature" we mean a temperature higher than the transition from the ordered (ferrimagnetic) phase to the disordered (paramagnetic) phase. This transition is the Curie temperature, $T_{\mathrm{C}}$, and it is an intrinsic property, depending entirely on the ferrite composition (except for ferrite nanoparticles, where the Curie temperature might depend on the nanoparticle size). The effects of temperature are shown in Fig. 4.1 for a polycrystalline NiZn ferrite with $x=0.65$. Most of spectra exhibit an additional absorption in the low field range $(H$ $<0.75 \mathrm{kOe}$ ). This non-resonant absorption is the low field microwave absorption (LFMA, Section 3) and will be discussed in Section 5. We focus now on the resonance phenomena.

The reported Curie temperature for this composition is $\sim 435 \mathrm{~K}$ (Globus et al 1977); the spectrum at $460 \mathrm{~K}$ corresponds therefore to the paramagnetic state. In these conditions, the thermal energy is high enough to overwhelm the internal field that results in the long range order of spins, and they are free to interact with the DC field, $H_{\mathrm{DC}}$, and the microwave field, $h_{\mathrm{AC}}$ In the Larmor relation (Eq. 3.6),

$$
\omega=\gamma H
$$


(where $\omega$ is the resonance frequency, $\gamma$ is the gyromagnetic factor), $H$ is the total field on the spins. In the absence of any internal field, $H$ is simply the external applied field $H_{\text {res. }}$ The microwave absorption at $460 \mathrm{~K}$, Fig. 4.1, appears as a narrow, symmetric line. This is an EPR (electron spin resonance) spectrum.

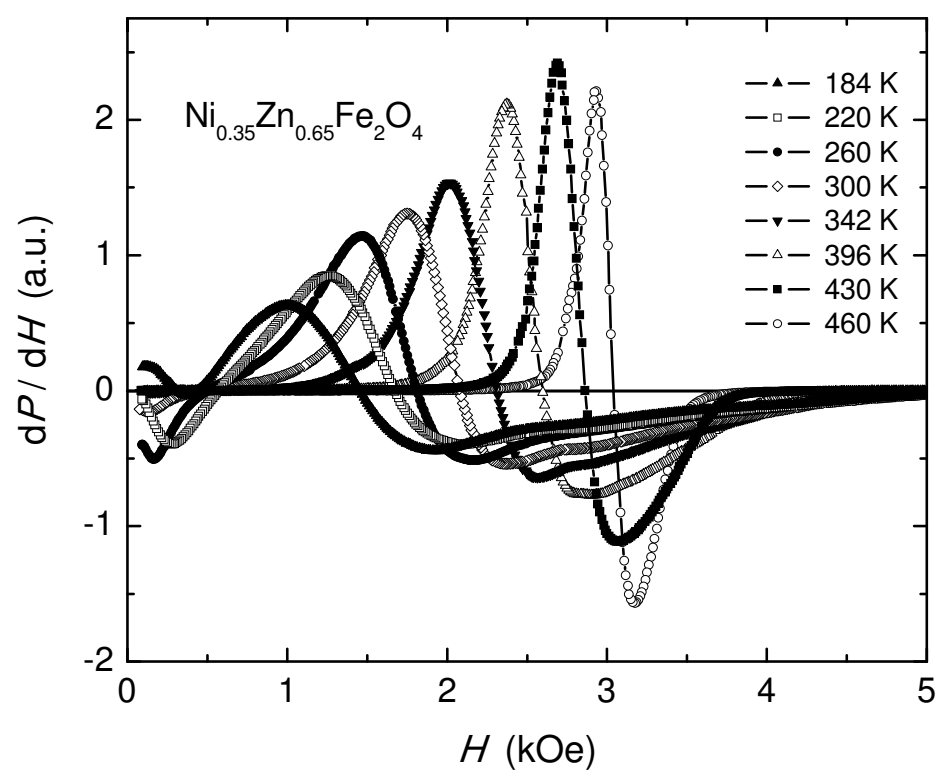

Fig. 4.1. Ferromagnetic resonance as a function of measuring temperature (184 $\leq T \leq 460 \mathrm{~K})$, on a polycrystalline ferrite with composition $\mathrm{Ni}_{0.35} \mathrm{Zn}_{0.65} \mathrm{Fe}_{2} \mathrm{O}_{4}$ (adapted from Montiel et al., 2004).

\subsection{Intermediate temperatures $\left(T<T_{C}\right)$}

As temperature decreases below the Curie transition, several changes are apparent in the resonance spectra. First, the resonance field (usually taken as the intercept of the line with the field axis) decreases. Second, the lineshape becomes broader as $T$ decreases, especially in the $H>H_{\text {res }}$ field region. These changes are due to the rise of the internal field, $H_{\mathrm{i}}$, leading to the long range order of magnetic moments. For $T<T_{\mathrm{C}}, H_{\mathrm{i}}$ possesses a larger energy than the thermal energy of the ferrite. Accordingly, the total field in the Larmor relation should include now the contribution from the internal field,

$$
H=H_{\text {res }}+H_{\mathrm{i}}
$$

The internal field is the combination of all the factors associated with the long range order in the ferrite: the exchange field, $H_{\mathrm{ex}}$, the anisotropy field, $H_{\mathrm{K}}$, the demagnetization field, $H_{\mathrm{d}}$, the porosity field, $H_{\mathrm{p}}$ (which is the field due to the appearance of magnetic dipoles on pores), etc. An additional source of inhomogeneity in ferrites is associated with differences, as well as with disorder, in site occupancy by the cations. As discussed in Section 2, transition metal cations have different stabilization energies on sites with diverse symmetry, as the tetrahedral and octahedral sites of spinels. While some of them exhibit a clear "preference" for one of the sites (i.e., $\mathrm{Zn}^{2+}$ for tetrahedral or A sites, $\mathrm{Co}^{2+}, \mathrm{Ni}^{2+}$, and $\mathrm{Fe}^{2+}$ for 
octahedral or B sites), other cations can be found equally on both sites $\left(\mathrm{Fe}^{3+}\right)$. To make things more complicated, it is possible to change the cation distribution by means of thermal treatments. The resonance phenomenon can be therefore slightly different when this occupancy of sites is not strictly homogeneous, since some terms of the internal field are not exactly the same for all the microwave absorbers.

The other source of inhomogeneity in the internal field is the disorder in the site occupancy. Even if the occupancy of sites is well determined (i.e., in Ni-Zn ferrite, all $\mathrm{Zn}$ cations on A sites, all $\mathrm{Ni}$ cations on $\mathrm{B}$ sites), there can be an inhomogeneous distribution of each of them on the sites. A simple example could be nickel ferrite, $\mathrm{NiFe}_{2} \mathrm{O}_{4}$, with all $\mathrm{Ni}^{2+}$ on $\mathrm{B}$ sites (and of course, $\mathrm{Fe}^{3+}$ on both sites). An extreme arrangement would be a long range order of $\mathrm{Ni}^{2+}$ and $\mathrm{Fe}^{3+}$ on octahedral sites; the cation nearest neighbor of any $\mathrm{Ni}^{2+}$ is then one $\mathrm{Ni}^{2+}$ and two $\mathrm{Fe}^{3+}$, and viceversa (see Fig. 2.2). On the other extreme, the "disordered" spinel would be the one with $\mathrm{Ni}^{2+}$ and $\mathrm{Fe}^{3+}$ randomly distributed on $\mathrm{B}$ sites. Obviously, the cation nearest neighbor of a given $\mathrm{Ni}^{2+}$ could be, on equal probability another $\mathrm{Ni}^{2+}$ or a $\mathrm{Fe}^{3+}$. Internal fields would not be strictly the same for each situation. These two sources of line broadening in FMR in ferrites depending on cation distribution could be written as $H_{\text {dist. }}$ To our knowledge, this contribution has not been discussed in literature.

The internal field can therefore be expressed as:

$$
H_{\mathrm{i}}=H_{\mathrm{ex}}+H_{\mathrm{K}}+H_{\mathrm{d}}+H_{\mathrm{p}}+H_{\mathrm{dis}}
$$

Figure 4.2 shows the behavior of the resonance field, $H_{\text {res, }}$ as a function of temperature. $H_{\text {res }}$ increases as temperature increases because the internal field decreases until it is overwhelmed by thermal vibrations at $T_{\mathrm{C}}$. For higher temperatures, the magnetic field needed to satisfy the Larmor relation has to be supplied entirely by the external field.

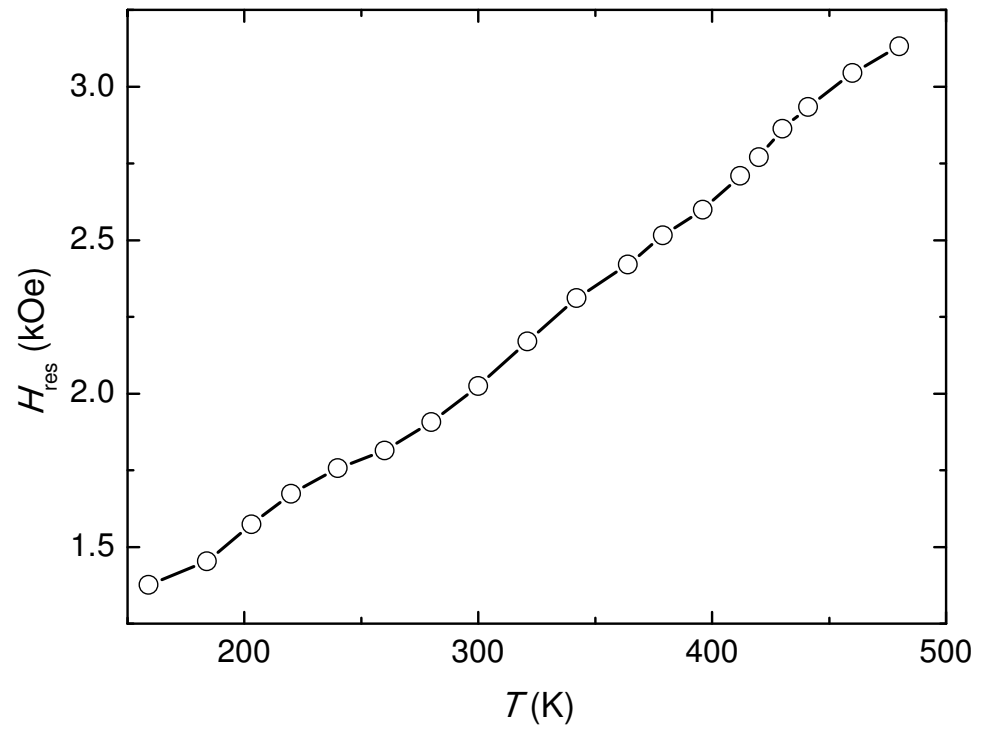

Fig. 4.2. Variation of the resonance field, $\mathrm{H}_{\text {res, }}$ with temperature for $\mathrm{Ni}_{0.35} \mathrm{Zn}_{0.65} \mathrm{Fe}_{2} \mathrm{O}_{4}$ ferrites (adapted from Alvarez et al, 2010). 
The total linewidth, $\Delta H$ (taken as the field between the maximum and the minimum in the resonance signal), has also an additive character in polycrystalline materials, and can be written:

$$
\Delta H=\Delta H_{\mathrm{p}}+\Delta H_{\mathrm{K}}+\Delta H_{\text {eddy }}+\Delta H_{\mathrm{d}}+\Delta H_{\text {dist }}
$$

Where $\Delta H_{\mathrm{p}}$ is the linewidth broadening associated with porosity, $\Delta H_{\mathrm{K}}$ is due to magnetic anisotropy, $\Delta H_{\text {eddy }}$ is related with eddy currents, $\Delta H_{\mathrm{d}}$ is the linewidth broadening produced by demagnetizing fields, and $\Delta H_{\text {dist }}$ is the linewidth broadening originated by variations in cation distribution on the A and B sites of ferrite. It appears that anisotropy, and in particular magnetocrystalline anisotropy has a strong contribution to total linewidth. By measuring nickel ferrite with $\mathrm{Co}^{2+}$ substitutions, Sirvetz \& Saunders (1956) observed a minimum in linewitdth for the composition corresponding to the compensation of anisotropies $(x=0.025$ in $\mathrm{Co}_{x} \mathrm{Ni}_{1-x} \mathrm{Fe}_{2} \mathrm{O}_{4}$ ), since nickel ferrite has a small negative contribution (single-ion contribution to anisotropy), while cobalt cations provide a strong positive contribution to the total magnetocrystalline anisotropy. More recently, Byun et al (2000) showed that in the case of Cosubstituted NiZnCu ferrites, $\Delta H$ increases for a Co composition higher than the magnetocrystalline anisotropy compensation point. Another source of linewidth broadening is certainly related with the polycrystalline nature of most samples. By modeling one ensemble of single domain nanoparticles, Sukhov et al (2008) have shown that the random distribution of anisotropy axis is directly associated with the broadening of the FMR signal.

Figure 4.3 shows the behavior of linewidth with temperature for the same sample than Figs. 4.2 and 4.1. A clear change in slope can be observed at about $430 \mathrm{~K}$, and a smooth variation is also apparent at about $250 \mathrm{~K}$. The former is associated with the Curie transition, which for this $\mathrm{Ni} / \mathrm{Zn}$ ratio is $\sim 430 \mathrm{~K}$ (Valenzuela, 2005a), and the latter with a change in magnetic structure which will be discussed later. By comparison with Fig. 4.2 it appears that linewidth, $\Delta H$, is more sensitive to structural changes than the resonance field, $H_{\text {res }}$.

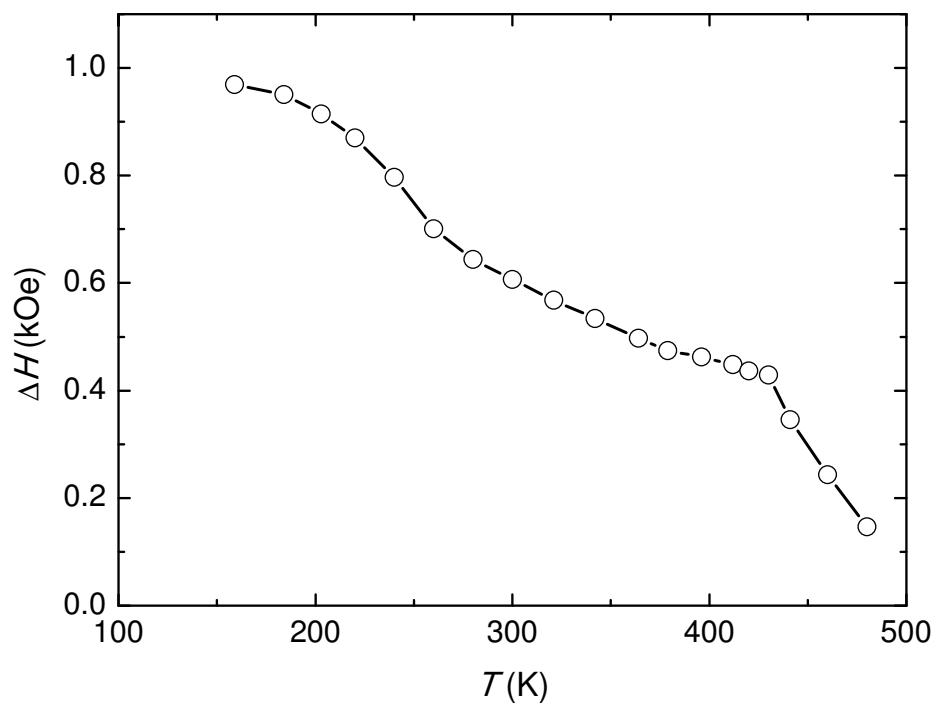

Fig. 4.3. Variations in linewidth with temperature, for $\mathrm{Ni}_{0.35} \mathrm{Zn}_{0.65} \mathrm{Fe}_{2} \mathrm{O}_{4}$ ferrites (adapted from Alvarez et al, 2010). 
The increase in resonance field as temperature rises is due to the fact that internal field decreases (exchange interaction, anisotropy field, and the fields associated with magnetization, i.e., demagnetization fields on surfaces including the ones created by porosity). In contrast, linewidth decreases with temperature, essentially because one of the major contributions to $\Delta H$ is originated by magnetocrystalline anisotropy, and this contribution is proportional to this parameter (Byun et al 2000). At $T>T_{\mathrm{C}}$, as discussed in Section 4.1, the resonance line becomes narrow and symmetrical, as the spectrum for $T=460 \mathrm{~K}$ in Fig. 4.1.

\subsection{Low temperatures $\left(T \ll T_{C}\right)$}

$\mathrm{Ni}-\mathrm{Zn}$ ferrites present other interesting phenomena at $T$ below room temperature. These appear as small "bumps" in the thermal behavior of both the resonance field and the linewidth, at about $240 \mathrm{~K}$. These phenomena are more evident in LFMA (Low-Field Microwave Absorption). In this absorption mode (see Section 3.), the system is far from the resonance conditions as stated in the Larmor relation, and it can be thought as the interaction between the microwave field and the ordered spins in the material as the magnetization state progresses from the demagnetized state toward magnetic saturation. In the simple case, LFMA appears as an antisymmetric signal at both sides of the $H=0$, Fig. 4.4. LFMA also exhibits hysteresis by cycling the application of the magnetic field.

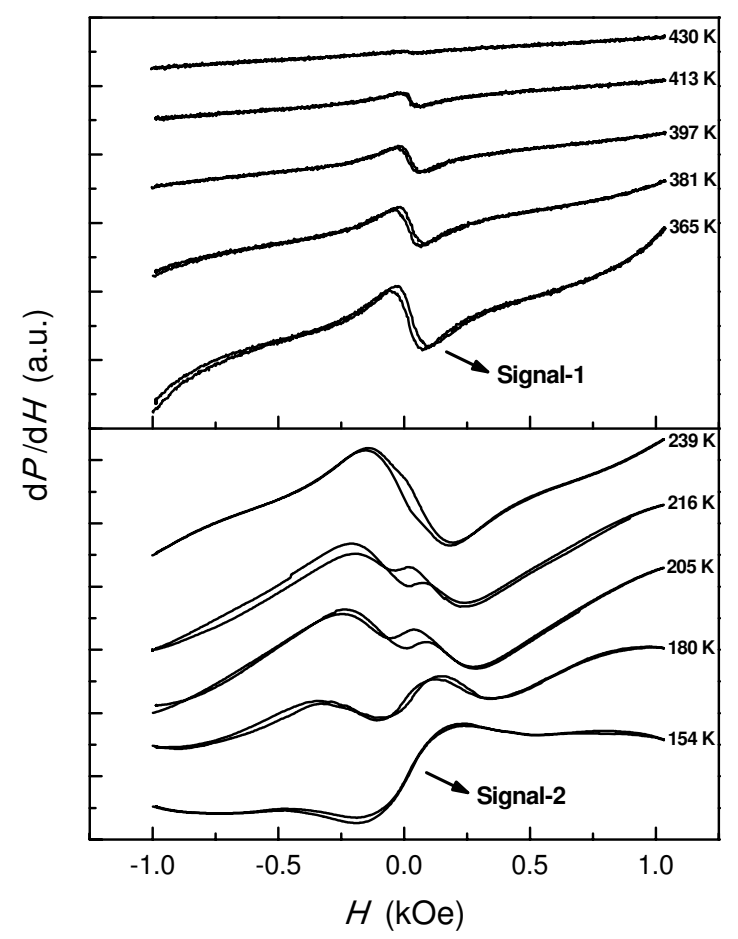

Fig. 4.4. LFMA measurements of $\mathrm{Ni}_{0.35} \mathrm{Zn}_{0.65} \mathrm{Fe}_{2} \mathrm{O}_{4}$ ferrites, for selected temperatures. The temperature range in the upper graph is $300-430 \mathrm{~K}$, while in the lower one it is 154-239 K (adapted from Alvarez et al 2010). 
Several features are significant in these plots. Beginning with the high temperatures (upper part of Fig. 4.4), it is evident that the amplitude of the signal at both sides of $H=0$ decreases as $T$ increases, leading to a flat response for $T \geq 430 \mathrm{~K}$, which is the Curie point. It is therefore confirmed that LFMA is associated with magnetization processes in the ordered phase.

Also, it can be observed that the field corresponding to the peak to peak magnetic field values, maxima (for negative fields) and the minima (for positive fields) increases as $T$ decreases. By comparing with a direct calculation of the anisotropy field, $H_{\mathrm{K}}$, Valenzuela et al (2011) were able to show that the amplitude between maxima-minima in LFMA is directly associated with $H_{\mathrm{K}}$, upper part of Fig. 4.5.

By a comparison between the two sets of curves separated by $T \sim 250 \mathrm{~K}$, it appears that there is a continuous evolution of the antisymmetrical signal, from high $T$ to low $T$, from a signal with the same phase as the FMR signal (Fig. 4.1) for $T>250 \mathrm{~K}$, to the opposite, also antisymmetric, but minimum-maximum (Min-Max), or out-of-phase signal, which is clearly reached at $T \leq 150$ $\mathrm{K}$. Both signals are centered on $H=0$. The presence of such out-of-phase signal has been correlated with the occurrence of a ferromagnetic ordering (Owens 2001, 2005). In fact, an-outof phase LFMA signal has been observed in many ferromagnetic systems (Montiel et al 2005, 2008, de Cos et al 2008). It can be assumed that a parallel, ferromagnetic arrangement of spins is related with this signal, while an antiparallel, ferrimagnetic structure leads to the opposite result (in phase signal). In the present case, the evolution of the signal when decreasing temperature should be associated with the appearance of a parallel arrangement of spins for $T$ $\leq 150 \mathrm{~K}$. Due to the Yafet-Kittel triangular structure, Fig. 2.5 (c), there is effectively a ferromagnetic arrangement simply by considering the components of the canted spins of cations on B sites. Figure 4.5 shows the evolution of Curie temperature and Yafet-Kittel transition for NiZn ferrites; the latter was determined by neutron diffraction (Satya Murthy et al, 1969). The results on Fig. 4.4 were obtained for $x=0.65$, leading to a Yafet-Kittel transition, $T_{Y K}$, about $250 \mathrm{~K}$, which is in very good agreement with these results.

The transition from the collinear arrangement to the Yafet-Kittel triangular structure can be detected (as temperature decreases) by means of MAMMAS experiments, as shown in Fig.

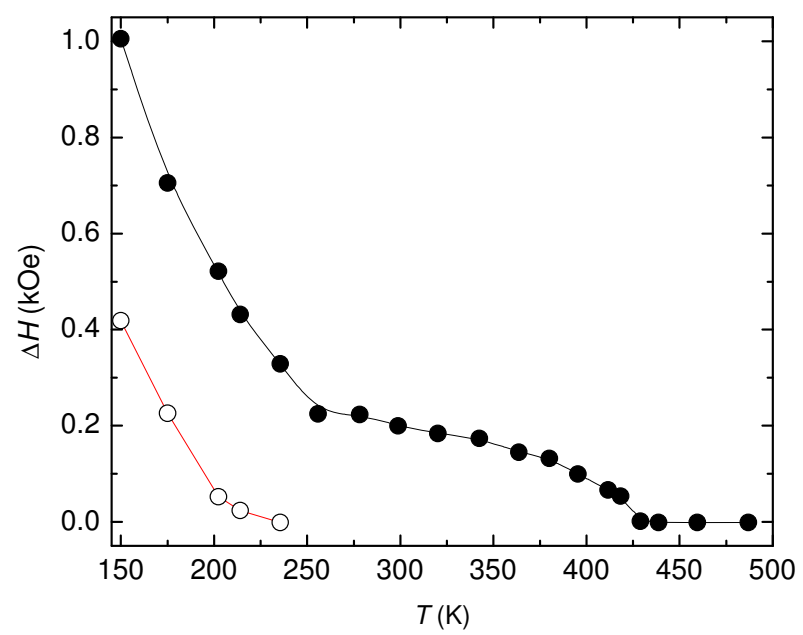

Fig. 4.5. Thermal variations of the peak-to-peak magnetic field of LFMA spectra for both signals. 


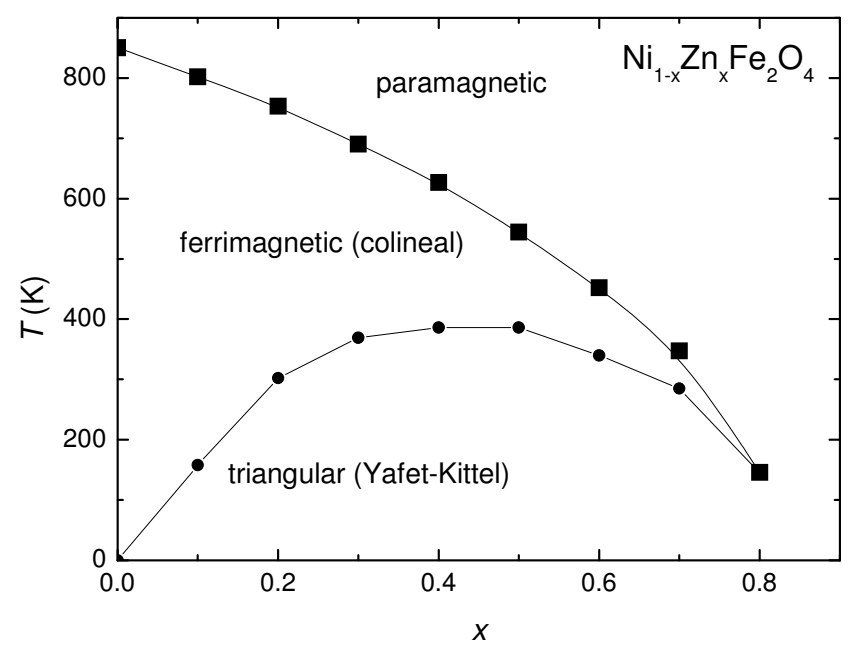

Fig. 4.6. Curie temperature and Yafet-Kittel temperature for NiZn ferrites (Adapted from Valenzuela 2005a and Satya Murthy et al 1969).

4.7. As explained in Section 3, the sample is subjected to a small magnetic field, and its microwave absorption is monitored as temperature is slowly changed. The MAMMAS response exhibits, from room temperature, a continuous decrease to a minimum value at about $240 \mathrm{~K}$. Then, the absorption increases again as the temperature keeps decreasing. These features point to a change in the microwave absorption regime due to a change in the material structure. In this case, all evidence is associated with the transition from the

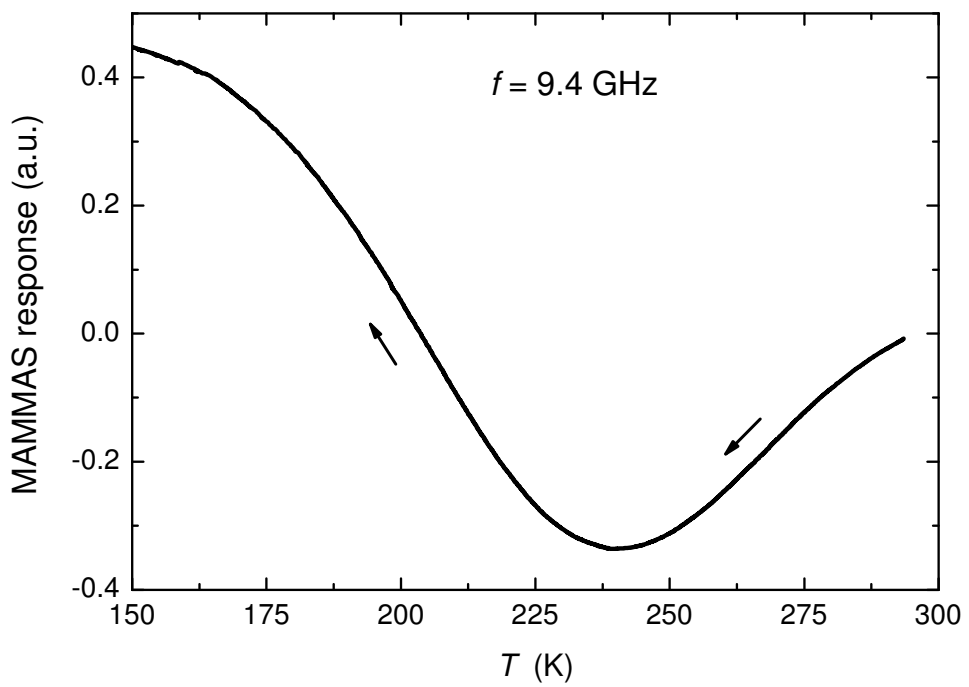

Fig. 4.7. Microwave absorption response of $\mathrm{Ni}_{0.35} \mathrm{Zn}_{0.65} \mathrm{Fe}_{2} \mathrm{O}_{4}$ ferrites in the MAMMAS experiment (Alvarez et al 2010). 
collinear ferromagnetic structure with iron in A sites of the spinel coupled by a superexchange interaction with iron cations (and nickel cations) on B sites, for $T>240 \mathrm{~K}$, to the triangular structure, where spins are no more collinear. Due to the weakening of the A$\mathrm{O}-\mathrm{B}$ interaction (as A sites become increasingly populated by zinc non magnetic ions), it becomes comparable to the B-O-B interaction which tends to establish an antiparallel geometry on the spins of ions on B sites.

\section{Conclusions}

As a conclusion, we can state that ferrites are complex materials: they offer a crystal complexity, with complex magnetic structures and complex magnetic properties. However, complexity can always be a rich source of knowledge. In addition to the well known ferromagnetic resonance methods, some significant steps can be done by investigating the microwave response of ferrites, particularly by using two novel research techniques, based on nonresonant absorption: low field and magnetically modulated microwave abosorption, which provide an original insight into these materials.

\section{Acknowledgments}

This work was partially supported by an ANR (France)-CONACyT (Mexico) grant (\#139292).

\section{References}

Alvarez G. \& Zamorano R. (2004). Characteristics of the magnetosensitive non-resonant power absorption of microwave by magnetic materials. Journal of Alloys and Compounds, Vol. 369, No. 1-2, (April 2004), pp. 231-234, ISSN: 0925-8388

Alvarez G., Montiel H., de Cos D., Zamorano R., García-Arribas A., Barandiaran J.M. \& Valenzuela R. (2007). Experimental and theoretical correlation between low-field power absorption and magnetoimpedance in amorphous materials. Journal of Non Crystalline Solids, Vol. 353, No. 8-10, (April 2007), pp. 902-904, ISSN: 0022-3093

Alvarez G., Montiel H., Barrón J.F., Gutierrez M.P., Zamorano R. (2010). Yafet-Kittel-type magnetic ordering in $\mathrm{Ni}_{0.35} \mathrm{Zn}_{0.65} \mathrm{Fe}_{2} \mathrm{O}_{4}$ ferrite detected by magnetosensitive microwave absorption measurements. Journal of Magnetism and Magnetic Materials Vol. 322 No. 3 (September 2009), pp. 348-352, ISSN: 0304-8853

Anderson P.W. (1959). New approach to the theory of superechange interactions. Physical Review, Vol. 115 No. 1 (February 1959) pp. 2-13, ISSN: 0031-899X

Byun T.Y., Byeon S.C., Hong K.S. and Kim C.K. Origin of line broadening in Co-substituted NiZnCu ferrites. (2000). Journal of Applied Physics Vol. 87 No. 9, (May 2000), pp. 6220-6222, ISSN: 0021-8979

Chiriac H., Colesniuc C.N. \& Ovari T.-A. (2000). FMR Investigation of the nanocrystalline FeCuNbSiB glass-covered wires. Journal of Magnetism and Magnetic Materials, Vol. 215-216, No. 1 (June 2000), pp. 407-409, ISSN: 0304-8853

De Cos D., García-Arribas A., Alvarez G., Montiel H., Zamorano R., Barandiaran J.M. \& Valenzuela R. (2007). Low field sensitivity for gigahertz magneto-impedance sensors. Sensor Letters Vol. 5, No. 1 (September 2007), pp. 73-76, ISSN: 1546-198X

Globus A., Pascard H., and Cagan V. (1977). Distance between ions and fundamental properties in ferrites. Journal de Physique, Vol. C1-38, (month 1977), pp. C1-163-C1168, ISSN: 0449-1947 
Hill R.J., Craig J.R. and Gibbs G.V. (1979). Systematics of the spinel structure type. Physics and Chemistry of Minerals, Vol. 4 (April 1979) pp. 317-339, ISSN: 0342-1791

Kittel C. (2005). Introduction to Solid State Physics, $8^{\text {th }}$ Edition, John Wiley and Sons, N.Y. ISBN: 0-471-41526-X

Medina A.N., Knobel M., Salem-Sugui S., \& Gandra F.G. (1999). Resonant microwave cavity response of amorphous ribbons. Journal of Applied Physics, Vol. 79, No. 8 (September 1999), pp. 5462-5464, ISSN: 1054-1500

Montiel H., Alvarez, G., Gutiérrez M.P., Zamorano, R., and Valenzuela R. (2004). Microwave absorption in Ni-Zn ferrites through the Curie Transition. Journal of Alloys and Compounds, Vol. 369, No. 1-2, (April 2004), pp. 141-143, ISSN: 0925-8388

Montiel H., Alvarez, G., Betancourt I., Zamorano, R., and Valenzuela R. (2005). Correlation between low-field microwave absorption and magnetoimpedance in Co-based amorphous ribbons. Applied Physics Letters, Vol. 86 No 7 (February 2005) paper 072503, pp. 1-3, ISSN: 0003-6951

Montiel H., Alvarez G., Gutiérrez M.P., Zamorano R. \& Valenzuela R. (2006). The effect of metal-to-glass ratio on the low-field microwave absorption at $9.4 \mathrm{GHz}$ of glasscoated CoFeBSi microwires. IEEE Transactions on Magnetics Vol. 42, No. 10, (September 2006), pp. 3380-3382 ISSN: 0018-9464

Owens F.J. (2001). Resonant and nonresonant microwave absorption study of ferromagnetic transition in $\mathrm{RuSr}_{2} \mathrm{Gd}_{0.5} \mathrm{Eu}_{0.5} \mathrm{Cu}_{2} \mathrm{O}_{8}$ superconductor. Physica C vol. 353 No. 3, 4 (May 2001), pp. 265-269, ISSN: 0921-4534

Owens F.J. (2005). Ferromagnetism above room temperature in bulk sintered gallium phosphide doped with manganese. Journal of Physics and Chemistry of Solids Vol. 66 No. 5 (May 2005), pp. 793-790, ISSN: 0022-3697

Pilbrow J.R. (1990). Transition ion electron paramagnetic resonance, Clarendon Press, Oxford. ISBN: 0-19-855214-9

Ravindranathan P. \& Patil K.C. (1987). Journal of Materials Science Vol. 22, No. 9, (September 1987), pp. 3261- 3264, ISSN: 0022-2461

Rivoire M. \& Suran G. (1995). Magnetization of thin films with in-plane uniaxial anisotropy studied by microwave absorption. Journal of Applied Physics, Vol. 78, No. 3 (***1995), pp. 1899-1905 , ISSN: 0021-8979

Satya Murthy N.S., Natera M.G., Youssef S.J., Begum R.J., Srivastava C.M. (1969). YafetKittel Angles in Zinc-Nickel Ferrites. Physical Review Vol. 181 No. 2 (May 1969), pp. 969-977, ISSN: 1098-0121

Sirvetz M.H. and Saunders J.H. (1956). Resonance widths in polycrystalline nickel-cobalt ferrites. Physical Review Vol. 102 No. 2 (April 1956), pp. 366-367, ISSN: 1098-0121

Valenzuela R. (2005a). Magnetic Ceramics, Cambridge University Press, Cambridge, UK (September 2005) ISBN: 0-521-01843-9

Valenzuela R., Montiel H., Gutierrez M.P., Betancourt I. (2005b) Characterization of soft ferromagnetic materials by inductance spectroscopy and magnetoimpedance, Journal of Magnetism and Magnetic Materials, Vol. 294 No. 2 (July 2005), pp. 239-244, ISSN: 0304-8853

Valenzuela R., Gutiérrez M.P., Vázquez G. \& Acevedo U. (2011). (To be published).

Wu K.H., Shin Y.M., Yang C.C., Wang G.P., \& Horng D.N. (2006). Materials Letters Vol. 60, No. $(* * 2006)$ pp. 2707- , ISSN:

Yafet Y. \& Kittel C. (1952) Antiferromagnetic Arrangements in Ferrites. Physical Review, Vol. 87, No. 2, (March 1952), pp. 290-294, ISSN: 1098-0121 


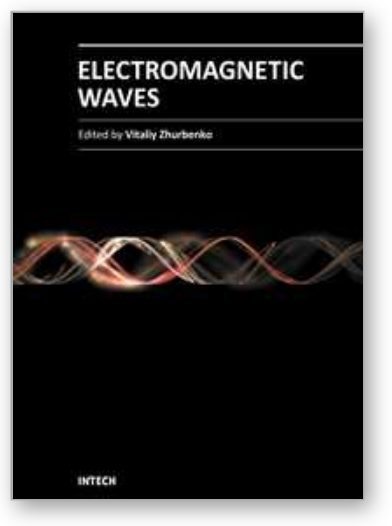

\author{
Electromagnetic Waves \\ Edited by Prof. Vitaliy Zhurbenko
}

ISBN 978-953-307-304-0

Hard cover, 510 pages

Publisher InTech

Published online 21, June, 2011

Published in print edition June, 2011

This book is dedicated to various aspects of electromagnetic wave theory and its applications in science and technology. The covered topics include the fundamental physics of electromagnetic waves, theory of electromagnetic wave propagation and scattering, methods of computational analysis, material characterization, electromagnetic properties of plasma, analysis and applications of periodic structures and waveguide components, and finally, the biological effects and medical applications of electromagnetic fields.

\title{
How to reference
}

In order to correctly reference this scholarly work, feel free to copy and paste the following:

Raul Valenzuela (2011). The Temperature Behavior of Resonant and Non-resonant Microwave Absorption in Ni-Zn Ferrites, Electromagnetic Waves, Prof. Vitaliy Zhurbenko (Ed.), ISBN: 978-953-307-304-0, InTech, Available from: http://www.intechopen.com/books/electromagnetic-waves/the-temperature-behavior-ofresonant-and-non-resonant-microwave-absorption-in-ni-zn-ferrites

\section{INTECH}

open science | open minds

\author{
InTech Europe \\ University Campus STeP Ri \\ Slavka Krautzeka 83/A \\ 51000 Rijeka, Croatia \\ Phone: +385 (51) 770447 \\ Fax: +385 (51) 686166 \\ www.intechopen.com
}

\author{
InTech China \\ Unit 405, Office Block, Hotel Equatorial Shanghai \\ No.65, Yan An Road (West), Shanghai, 200040, China \\ 中国上海市延安西路65号上海国际贵都大饭店办公楼 405 单元 \\ Phone: +86-21-62489820 \\ Fax: $+86-21-62489821$
}


(C) 2011 The Author(s). Licensee IntechOpen. This chapter is distributed under the terms of the Creative Commons Attribution-NonCommercialShareAlike-3.0 License, which permits use, distribution and reproduction for non-commercial purposes, provided the original is properly cited and derivative works building on this content are distributed under the same license. 